Research Article

\title{
Transmission Dynamics of Fractional Order Brucellosis Model Using Caputo-Fabrizio Operator
}

\author{
Olumuyiwa James Peter \\ Department of Mathematics, University of Ilorin, Ilorin, Kwara State, Nigeria \\ Correspondence should be addressed to Olumuyiwa James Peter; peterjames4real@gmail.com
}

Received 10 June 2020; Accepted 27 July 2020; Published 14 August 2020

Academic Editor: Jaume Giné

Copyright ( $\odot 2020$ Olumuyiwa James Peter. This is an open access article distributed under the Creative Commons Attribution License, which permits unrestricted use, distribution, and reproduction in any medium, provided the original work is properly cited.

In this paper, a noninteger order Brucellosis model is developed by employing the Caputo-Fabrizio noninteger order operator. The approach of noninteger order calculus is quite new for such a biological phenomenon. We establish the existence, uniqueness, and stability conditions for the model via the fixed-point theory. The initial approachable approximate solutions are derived for the proposed model by applying the iterative Laplace transform technique. Finally, numerical simulations are conducted for the analytical results to visualize the effect of various parameters that govern the dynamics of infection, and the results are presented using plots.

\section{Introduction}

Brucellosis is a contagious zoonotic infection that is attributable to diverse species of Brucella. Although the disease is well contained in Australia and the UK, the annual global incidence of Brucellosis is estimated above $500000[1,2]$. Brucellosis is endemic in the Caribbean, South America, Mediterranean Europe, Africa, Asia, and in the Middle East and poses considerable economic consequences in some of the regions despite the enforcement of firm veterinary hygiene interventions [3]. Typically, animal Brucellosis is attributed to either direct or indirect contacts with the Brucellosis bacteria [4]. While direct contact is from contact with the infected secretions, droppings, or vertical transmission from mother-to-offspring during delivery, indirect contact occurs through exposure to contaminated environment or objects. It may also occur through respiratory or digestive tracks especially during feeding [5]. Human Brucellosis, on the contrary, is triggered by contamination from infectious animals or their products. Orchitis, epididymitis, placentitis, and abortions are the commonest consequences of Brucellosis [6]. The primary hosts for
Brucellosis are sheep, goats, cattle, buffalo, swine, horses, dogs, and man [1]. Control strategies for Brucella within domestic animals involve detection, inoculation, and elimination of the affected livestock [7]. However, rapid control of Brucellosis is achieved by the combination of the interventions.

The quantification of intricate and complex biological systems in terms of mathematical structures has posed a serious challenge to contemporary scientists worldwide. A good number of models with soliton solutions that are designed in terms of integer-order derivatives had been extensively studied in the last two decades [8-12]. Some of these models in the literature include Tzizeica-type equation [13], Fokas-Lenells equation [14], complex Ginzburg-Landau equation [15], differential-difference equation [16], nonlinear oscillator with discontinuities [17], and Bernstein wavelet equations [18]. The aforementioned models play significant roles in advancing suitable control procedures in a biological system [19].

Scientific and technical models of Brucellosis dynamics can be used to interpret the primary outcomes and condense the discoveries to the best advantage of protection at various 
stages; still, a number of those models in the form of nonfractional derivative were thoroughly investigated [20-23]. Researchers in mathematics have been engaged in the last decade in the formulation of mathematical models of Brucellosis to investigate the stability and dynamical behaviours of the disease $[24,25]$. The primary model of these kinds, which examine the indirect transmission mechanism of Brucellosis with the influence of immunology, was jointly investigated by Zhang et al. [26]. In [1], Li et al. proposed a model that quantified the spread of Brucellosis from sheep to man and among sheep. Unlike in [1], Brucellosis models that described the transmission of Brucellosis in the cattle population and that characterized stillbirths in dairy cattle are designed and analyzed by Tumwiine and Robert [27] and Nie et al. [6], respectively.

As time goes by, advancing mathematical models using noninteger order became a significant area of study since the evolution-related realities and evidence are represented more effectively in terms of noninteger calculus $[28,29]$. The elaborate investigations of such models play an exemplary role through advancing implementations of various real-life problem. A large number of logical and numerical procedures and schemes are proposed to define arbitrary and real solutions with noninteger operators for differential equations have been playing an exceptional task in the advancement of implementations of numerous problems. A good number of logical and numerical procedures and schemes are proposed to figure out indefinite and actual solutions of the differential equations with noninteger operators [30-35]. However, up till now, there is no work which has been designed to analyze the dynamics of Brucellosis with the fractional-order calculus.

There are four compartments into which the Brucellosis model in [26] is separated, denoted as susceptible dairy cows $S(t)$, the exposed dairy cow $E(t)$, infectious dairy cows $I(t)$, and the Brucella contaminated environment $B(t)$. The sum of the population at any time $t$ is represented by $N=S+E+I+B$. In [26], Zhang et al. proposed a Brucellosis model and analyzed the qualitative characteristics of the suggested model which is denoted by

$$
\begin{aligned}
S & =\theta+p(S+E+I)-\alpha S I-d S-\beta B S, \\
E & =\alpha S I+\beta B S-d E-\delta E, \\
I & =\delta E-d I-a I, \\
B & =n(E+I)-(\omega+c h) B,
\end{aligned}
$$

where $\theta$ denotes the yearly recruitment rate of dairy cows, the yearly birth rate of cow is $p$, the annual culling rate is $d$, the treatment outcome rate is $\delta$, the rate of yearly elimination for the positive dairy cows is $a$, the yearly number of Brucella is $n$, the yearly rate of natural death of Brucella is $\omega$, the rate of sterilization in a disinfection is $c$, and the yearly number of disinfections is $h$, and $\alpha$ is the indirect transmission rate and $\beta$ is the pathogen density-dependent component.

Motivated by the previously mentioned literature, we have applied Caputo-Fabrizio fractional order $\phi$, where $\phi \in(0,1)$ to explore dynamics of the Brucellosis model discussed in Zhang et al. [26]:

$$
\begin{aligned}
& { }^{\mathrm{CF}} D_{t}^{\phi} S(t)=\theta+p(S+E+I)-\alpha S I-d S-\beta B S, \\
& { }^{\mathrm{CF}} D_{t}^{\phi} E(t)=\alpha S I+\beta B S-d E-\delta E, \\
& { }^{\mathrm{CF}} D_{t}^{\phi} I(t)=\delta E-d I-a I, \\
& { }^{\mathrm{CF}} D_{t}^{\phi} B(t)=n(E+I)-(\omega+c h) B,
\end{aligned}
$$

with initial conditions $S(0)=b_{1}, E(0)=b_{2}, I(0)=b_{3}$, and $B(0)=b_{4}$.

The typical model in [26] is derived for $\phi=1$. As the Caputo-Fabrizio fractional order quantifies the previously mentioned phenomenon accurately. The numerical results are obtained by employing the Laplace transform method. Arbitrary values are allocated to the initial conditions and parameters to validate our results.

The remainder of this script is arranged as follows. In Section 2, some fundamental remarks and definitions associated with noninteger calculus are stated. In Section 3, existence and uniqueness conditions are derived by using the fixed-point theory. In Section 4, stability results are obtained via iterative Laplace transform technique. In Section 5, the proposed method is employed to noninteger order Brucellosis model and the results of the simulations are illustrated graphically. Section 6 is devoted to the discussions of numerical results. Finally, the conclusion of the work is stated in Section 7.

\section{Preliminaries}

Definition 1 (see [36]). Let $u_{1} \in H^{\prime}(0, a), a>0,0<\phi<1$, then the Caputo fractional operator is defined as

$$
{ }^{\mathrm{CF}} D_{t}^{\phi} u_{1}(t)=\frac{(2-\phi) M(\phi)}{2(1-\phi)} \int_{0}^{t} \exp \left[-\frac{\phi(t-S)}{1-\phi}\right] u_{1}^{\prime}(t) \mathrm{d} t,
$$

where $M(\phi)$ is the normalization function which depends on $\phi$, such that $M(\phi)=M(1)=1$.

Definition 2 (see [37]). The Caputo-Fabrizio fractional integral operator of order $0<\phi<1$ is defined as

$$
{ }^{\mathrm{CF}} J_{t}^{\phi} u_{1}(t)=\frac{(2-\phi) M(\phi)}{2(1-\phi)} u_{1}(t)+\frac{2 \phi}{(2-\phi) M(\phi)} \int_{0}^{t} u_{1}(t) \mathrm{d} t .
$$

Definition 3 (see [36]). The Laplace transformation for the Caputo fractional operator of order $0<\phi \leq 1$ and $n \in M$ is expressed as

$$
\begin{aligned}
L\left({ }^{\mathrm{CF}} J_{t}^{n+\phi} u_{1}(t)\right)(\rho) & =\frac{1}{1-\phi} L\left(u^{(n+1)}\right) L\left(\exp \left(-\frac{\phi}{1-\phi} t\right)\right) \\
& =\frac{\rho^{n+1} L\left(\left(u_{1}\right)\right)-\rho^{n} u_{1}(0)-\rho^{n-1} u_{1}^{\prime}(0) \ldots u_{1}^{(n)}(0)}{\rho+\phi(1-\rho)} .
\end{aligned}
$$


Generally, we have

$$
\begin{aligned}
L\left({ }^{\mathrm{CF}} D_{t}^{\phi} u(t)\right)(\rho) & =\frac{\rho L\left(u_{1}(t)\right)}{\rho+\phi(1-\rho)}, \quad n=0, \\
L\left({ }^{\mathrm{CF}} D_{t}^{\phi+1} u_{1}(t)\right)(\rho) & =\frac{\rho^{2} L\left(\left(u_{1}(t)\right)\right)-\rho u_{1}(0)-u_{1}^{\prime}(0)}{\rho+\phi(1-\rho)}, \quad n=1 .
\end{aligned}
$$

(6)

\section{Existence and Uniqueness}

This section deals with the existence and uniqueness of the fractional Brucellosis model using fixed-point theory [38-45]. By applying the fractional integral operator (2) into (4), we have

$$
\begin{aligned}
S(t)= & S(0)+\frac{2(1-\phi)}{(2 \phi) M(\phi)}(\theta+p(S(t)+E(t)+I(t))-\alpha S(t) I(t)-d S(t)-\beta B(t) S(t))+ \\
& \cdot \frac{2(1-\phi)}{(2 \phi) M(\phi)} \int_{0}^{t}(\theta-p(S(\varepsilon)+E(\varepsilon)+I(\varepsilon))-\alpha S(\varepsilon) I(t)-d S(\varepsilon)-d S(\varepsilon)-\beta B(\varepsilon) S(\varepsilon)) \\
E(t)= & E(0)+\frac{2(1-\phi)}{(2 \phi) M(\phi)}(\alpha S(t) I(t)+\beta B(t) S(t)-d E(t)-\delta E(t))+ \\
& \cdot \frac{2(1-\phi)}{(2 \phi) M(\phi)} \int_{0}^{t}(\alpha S(\varepsilon) I(\varepsilon)+\beta B(\varepsilon) S(\varepsilon)-d E(\varepsilon)-\delta E(\varepsilon)) d \varepsilon \\
I(t)= & I(0)+\frac{2(1-\phi)}{(2 \phi) M(\phi)}(\delta E(t)-d I(t)-a I(t))+\frac{2(1-\phi)}{(2 \phi) M(\phi)} \int_{0}^{t}(\delta E(\varepsilon)-d I(\varepsilon)-a I(\varepsilon)) d \varepsilon \\
B(t)= & B(0)+\frac{2(1-\phi)}{(2 \phi) M(\phi)}(n(E(t)+I(t))-(\omega+c) B(t))+\frac{2(1-\phi)}{(2 \phi) M(\phi)} \int_{0}^{t}(n(E(\varepsilon)+I(\varepsilon)-(\omega+c h)) B(\varepsilon)) d \varepsilon
\end{aligned}
$$

For clarity, we consider the following kernels:

$$
\begin{aligned}
& \phi_{1}=\theta+p(S+E+I)-\alpha S I-d S-\beta B S, \\
& \phi_{2}=\alpha S I+\beta B S-d E-\delta E, \\
& \phi_{3}=\delta E-d I-a I, \\
& \phi_{4}=n(E+I)-(\omega+c h) B .
\end{aligned}
$$

Proof. Let $S_{1}$ and $S_{2}$ for the kernel given $\phi_{1}, E_{1}$ and $E_{2}$ for the kernel $\phi_{2}, I_{1}$ and $I_{2}$ for the kernel $\phi_{3}$, and $B_{1}$ and $B_{2}$ for the kernel $\phi_{4}$ be the respective functions associated with the following:

$$
\begin{aligned}
\left\|\phi_{1}\left(t, S_{1}(t)\right)-\phi_{1}\left(t, S_{2}(t)\right)\right\| & =\left\|-(p+\alpha I+d+\beta B)\left(S_{1}(t)-S_{2}(t)\right)\right\|, \\
\left\|\phi_{2}\left(t, E_{1}(t)\right)-\phi_{2}\left(t, E_{2}(t)\right)\right\| & =\left\|-(d+\delta)\left(E_{1}(t)-E_{2}(t)\right)\right\|, \\
\left\|\phi_{3}\left(t, I_{1}(t)\right)-\phi_{1}\left(t, I_{2}(t)\right)\right\| & =\left\|-(d+a)\left(I_{1}(t)-I_{2}(t)\right)\right\|, \\
\left\|\phi_{4}\left(t, B_{1}(t)\right)-\phi_{4}\left(t, B_{2}(t)\right)\right\| & =\left\|-(\omega+c h)\left(B_{1}(t)-B_{2}(t)\right)\right\| .
\end{aligned}
$$

By applying the properties of the norm on (10), respectively, we have 


$$
\begin{aligned}
\left\|\phi_{1}\left(t, S_{1}(t)\right)-\phi_{1}\left(t, S_{2}(t)\right)\right\| & =\left\|-(p+\alpha I+d+\beta B)\left(S_{1}(t)-S_{2}(t)\right)\right\| \\
& \leq(\alpha\|I\|+\beta\|B\|)\left\|S_{1}(t)-S_{2}(t)\right\| \\
& \leq a_{1}\left\|S_{1}(t)-S_{2}(t)\right\|,
\end{aligned}
$$

where $a_{1}=\alpha_{i}+\beta b, i=\max _{t \in \Omega}\|I(t)\|, b=\max _{t \in \Omega}\|B(t)\|$, are bounded functions. Subsequently, we have

$$
\begin{aligned}
\left\|\phi_{2}\left(t, E_{1}(t)\right)-\phi_{1}\left(t, E_{2}(t)\right)\right\| & =\leq a_{2}\left\|E_{1}(t)-E_{2}(t)\right\|, \\
\left\|\phi_{3}\left(t, I_{1}(t)\right)-\phi_{3}\left(t, I_{2}(t)\right)\right\| & =\leq a_{3}\left\|I_{1}(t)-I_{2}(t)\right\|, \\
\left\|\phi_{4}\left(t, B_{1}(t)\right)-\phi_{4}\left(t, B_{2}(t)\right)\right\| & =\leq a_{4}\left\|B_{1}(t)-B_{2}(t)\right\|,
\end{aligned}
$$

where $a_{2}=(d+\delta), a_{3}=(d+a)$, and $a_{4}=(\omega+c h)$.

We construct the following recursive formula:

$$
\begin{aligned}
& S_{n}(t)=\frac{2(1-\phi)}{(2-\phi) M(\phi)} \phi_{1}\left(t, S_{n-1}(t)\right)+\frac{2 \phi}{(2-\phi) M(\phi)} \int_{0}^{t} \phi_{1}\left(\varepsilon, S_{n-1}(\varepsilon)\right) \mathrm{d} \varepsilon, \\
& E_{n}(t)=\frac{2(1-\phi)}{(2-\phi) M(\phi)} \phi_{2}\left(t, E_{n-1}(t)\right)+\frac{2 \phi}{(2-\phi) M(\phi)} \int_{0}^{t} \phi_{1}\left(\varepsilon, E_{n-1}(\varepsilon)\right) \mathrm{d} \varepsilon, \\
& I_{n}(t)=\frac{2(1-\phi)}{(2-\phi) M(\phi)} \phi_{3}\left(t, I_{n-1}(t)\right)+\frac{2 \phi}{(2-\phi) M(\phi)} \int_{0}^{t} \phi_{1}\left(\varepsilon, I_{n-1}(\varepsilon)\right) \mathrm{d} \varepsilon, \\
& B_{n}(t)=\frac{2(1-\phi)}{(2-\phi) M(\phi)} \phi_{4}\left(t, B_{n-1}(t)\right)+\frac{2 \phi}{(2-\phi) M(\phi)} \int_{0}^{t} \phi_{1}\left(\varepsilon, B_{n-1}(\varepsilon)\right) \mathrm{d} \varepsilon .
\end{aligned}
$$

Furthermore, by applying triangular inequality, we have

$$
\begin{aligned}
\left\|D_{n}(t)\right\|= & \left\|S_{n}(t)-S_{n-1}(t)\right\| \leq \frac{2(1-\phi)}{(2-\phi) M(\phi)}\left\|\phi_{1}\left(t, S_{n-1}(t)\right)-\phi_{1}\left(t, S_{n-2}(t)\right)\right\| \\
& +\frac{2 \phi}{(2-\phi) M(\phi)}\left\|\left[\int_{0}^{t} \phi_{1}\left(\varepsilon, S_{n-1}(\varepsilon)\right)-\phi_{1}\left(\varepsilon, S_{n-2}(\varepsilon)\right)\right] \mathrm{d} \varepsilon\right\|, \\
\left\|F_{n}(t)\right\|= & \left\|E_{n}(t)-E_{n-1}(t)\right\| \leq \frac{2(1-\phi)}{(2-\phi) M(\phi)}\left\|\phi_{2}\left(t, E_{n-1}(t)\right)-\phi_{2}\left(t, E_{n-2}(t)\right)\right\| \\
& +\frac{2 \phi}{(2-\phi) M(\phi)}\left\|\left[\int_{0}^{t} \phi_{2}\left(\varepsilon, E_{n-1}(\varepsilon)\right)-\phi_{2}\left(\varepsilon, E_{n-2}(\varepsilon)\right)\right] \mathrm{d} \varepsilon\right\|, \\
\left\|I_{n}(t)\right\|= & \left\|I_{n}(t)-I_{n-1}(t)\right\| \leq \frac{2(1-\phi)}{(2-\phi) M(\phi)}\left\|\phi_{3}\left(t, I_{n-1}(t)\right)-\phi_{3}\left(t, I_{n-2}(t)\right)\right\| \\
& +\frac{2 \phi}{(2-\phi) M(\phi)}\left\|\left[\int_{0}^{t} \phi_{3}\left(\varepsilon, I_{n-1}(\varepsilon)\right)-\phi_{1}\left(\varepsilon, I_{n-2}(\varepsilon)\right)\right] \mathrm{d} \varepsilon\right\|, \\
\left\|B_{n}(t)\right\|= & \left\|B_{n}(t)-B_{n-1}(t)\right\| \leq \frac{2(1-\phi)}{(2-\phi) M(\phi)}\left\|\phi_{4}\left(t, B_{n-1}(t)\right)-\phi_{4}\left(t, B_{n-2}(t)\right)\right\| \\
& +\frac{2 \phi}{(2-\phi) M(\phi)}\left\|\left[\int_{0}^{t} \phi_{4}\left(\varepsilon, B_{n-1}(\varepsilon)\right)-\phi_{4}\left(\varepsilon, B_{n-2}(\varepsilon)\right)\right] \mathrm{d} \varepsilon\right\| .
\end{aligned}
$$

$S_{n}(t)=\sum_{m=0}^{\infty} D_{m}(t), I_{n}(t)=\sum_{m=0}^{\infty} F_{m}(t), I_{n}(t)=\sum_{m=0}^{\infty}$ $G_{m}(t)$, and $B_{n}(t)=\sum_{m=0}^{\infty} H_{m}(t)$.
The kernels $\phi_{1}, \phi_{2}, \phi_{3}$, and $\phi_{4}$ which satisfy the Lipschitz condition are therefore obtained: 


$$
\begin{aligned}
& \left\|D_{n}(t)\right\|=\left\|S_{n}(t)-S_{n-1}(t)\right\| \leq \frac{2(1-\phi)}{(2-\phi) M(\phi)} a_{1}\left\|S_{n-1}(t)-S_{n-2}(t)\right\|+\frac{2 \phi}{(2-\phi) M(\phi)} a_{1} \int_{0}^{t}\left\|S_{n-1}(\varepsilon)-S_{n-2}(\varepsilon)\right\| \mathrm{d} \varepsilon, \\
& \left\|F_{n}(t)\right\|=\left\|E_{n}(t)-E_{n-1}(t)\right\| \leq \frac{2(1-\phi)}{(2-\phi) M(\phi)} a_{2}\left\|E_{n-1}(t)-E_{n-2}(t)\right\|+\frac{2 \phi}{(2-\phi) M(\phi)} a_{2} \int_{0}^{t}\left\|E_{n-1}(\varepsilon)-E_{n-2}(\varepsilon)\right\| \mathrm{d} \varepsilon, \\
& \left\|G_{n}(t)\right\|=\left\|I_{n}(t)-I_{n-1}(t)\right\| \leq \frac{2(1-\phi)}{(2-\phi) M(\phi)} a_{3}\left\|I_{n-1}(t)-I_{n-2}(t)\right\|+\frac{2 \phi}{(2-\phi) M(\phi)} a_{3} \int_{0}^{t}\left\|I_{n-1}(\varepsilon)-I_{n-2}(\varepsilon)\right\| \mathrm{d} \varepsilon, \\
& \left\|H_{n}(t)\right\|=\left\|B_{n}(t)-B_{n-1}(t)\right\| \leq \frac{2(1-\phi)}{(2-\phi) M(\phi)} a_{4}\left\|B_{n-1}(t)-B_{n-2}(t)\right\|+\frac{2 \phi}{(2-\phi) M(\phi)} a_{4} \int_{0}^{t}\left\|B_{n-1}(\varepsilon)-B_{n-2}(\varepsilon)\right\| \mathrm{d} \varepsilon .
\end{aligned}
$$

This proves the result.

Proof. Following the result in (15) and with respect to the

Theorem 2. The SEIB Brucellosis model involving the fracrecursive expression, we get the following: tional operator in (2) has a solution (existence of the solution).

$$
\begin{aligned}
& \left\|D_{n}(t)\right\| \leq\|S(0)\|+\left[\left(\frac{2(1-\phi)}{(2-\phi) M(\phi)} a_{1}\right)^{n}+\left(\frac{2 \phi}{(2-\phi) M(\phi)} a_{1}(t)\right)^{n}\right], \\
& \left\|F_{n}(t)\right\| \leq\|E(0)\|+\left[\left(\frac{2(1-\phi)}{(2-\phi) M(\phi)} a_{2}\right)^{n}+\left(\frac{2 \phi}{(2-\phi) M(\phi)} a_{2}(t)\right)^{n}\right], \\
& \left\|G_{n}(t)\right\| \leq\|I(0)\|+\left[\left(\frac{2(1-\phi)}{(2-\phi) M(\phi)} a_{3}\right)^{n}+\left(\frac{2 \phi}{(2-\phi) M(\phi)} a_{3}(t)\right)^{n}\right], \\
& \left\|H_{n}(t)\right\| \leq\|B(0)\|+\left[\left(\frac{2(1-\phi)}{(2-\phi) M(\phi)} a_{4}\right)^{n}+\left(\frac{2 \phi}{(2-\phi) M(\phi)} a_{4}(t)\right)^{n}\right] .
\end{aligned}
$$

Hence, (16) exists, additionally, we show that (16) is the solution of (2) with the following assumption:

$$
\begin{aligned}
& S(t)=S_{n}(t)-\Delta_{1(n)}(t), \\
& E(t)=E_{n}(t)-\Delta_{2(n)}(t), \\
& I(t)=I_{n}(t)-\Delta_{3(n)}(t), \\
& B(t)=B_{n}(t)-\Delta_{4(n)}(t),
\end{aligned}
$$

where the remaining terms of the solution are $\Delta_{1(n)}, \Delta_{2(n)}, \Delta_{3(n)}$, and $\Delta_{4(n)}$. Hence, we have

$$
\begin{aligned}
& S_{n}(t)-S_{n-1}(t)=\frac{2(1-\phi)}{(2-\phi) M(\phi)} \phi_{1}\left(t, S(t)-\Delta_{1(n)}(t)\right)+\frac{2 \phi}{(2-\phi) M(\phi)} \int_{0}^{t} \phi_{1}\left(\varepsilon, S(\varepsilon)-\Delta_{1(n)}(\varepsilon)\right) \mathrm{d} \varepsilon, \\
& E_{n}(t)-E_{n-1}(t)=\frac{2(1-\phi)}{(2-\phi) M(\phi)} \phi_{2}\left(t, E(t)-\Delta_{2(n)}(t)\right)+\frac{2 \phi}{(2-\phi) M(\phi)} \int_{0}^{t} \phi_{2}\left(\varepsilon, E(\varepsilon)-\Delta_{2(n)}(\varepsilon)\right) \mathrm{d} \varepsilon, \\
& I_{n}(t)-I_{n-1}(t)=\frac{2(1-\phi)}{(2-\phi) M(\phi)} \phi_{3}\left(t, I(t)-\Delta_{3(n)}(t)\right)+\frac{2 \phi}{(2-\phi) M(\phi)} \int_{0}^{t} \phi_{3}\left(\varepsilon, I(\varepsilon)-\Delta_{3(n)}(\varepsilon)\right) \mathrm{d} \varepsilon, \\
& B_{n}(t)-B_{n-1}(t)=\frac{2(1-\phi)}{(2-\phi) M(\phi)} \phi_{4}\left(t, B(t)-\Delta_{4(n)}(t)\right)+\frac{2 \phi}{(2-\phi) M(\phi)} \int_{0}^{t} \phi_{4}\left(\varepsilon, B(\varepsilon)-\Delta_{4(n)}(\varepsilon)\right) \mathrm{d} \varepsilon .
\end{aligned}
$$


We make use of norm and Lipschitz condition to obtain $\| S(t)-S(0)-\frac{2(1-\phi)}{(2-\phi) M(\phi)} \phi_{1}\left(t, S(t)-\Delta_{1(n)}(t)\right)$

$$
\begin{gathered}
-\frac{2 \phi}{(2-\phi) M(\phi)} \int_{0}^{t} \phi_{1}\left(\varepsilon, S(\varepsilon)-\Delta_{1(n)}(\varepsilon)\right) \mathrm{d} \varepsilon\|\leq\| \Delta_{1(n)} \| \\
\left\{1+\left(\frac{2(1-\phi)}{(2-\phi) M(\phi)} a_{1}\right)+\left(\frac{2 \phi}{(2-\phi) M(\phi)} a_{1}(t)\right)\right\}
\end{gathered}
$$

Using limit $n \longrightarrow \infty$ in (19), we have

$$
\left\|\Delta_{1(n)}(t)\right\| \longrightarrow 0 \text {. }
$$

Therefore,

$$
\begin{aligned}
S(t)= & S(0)+\frac{2(1-\phi)}{(2-\phi) M(\phi)} \phi_{1}(t, S(t)) \\
& +\frac{2 \phi}{(2-\phi) M(\phi)} \int_{0}^{t} \phi_{1}(\varepsilon, S(\varepsilon)) \mathrm{d} \varepsilon .
\end{aligned}
$$

In the same way, as $n \longrightarrow \infty$, we obtain the following. Using the same approach, in (21), we have

$$
\begin{aligned}
& \left\|\Delta_{2(n)}(t)\right\| \longrightarrow 0, \\
& \left\|\Delta_{3(n)}(t)\right\| \longrightarrow 0, \\
& \left\|\Delta_{4(n)}(t)\right\| \longrightarrow 0 .
\end{aligned}
$$

Similarly, from (21), there exists a solution of (2).

Theorem 3. The SEIB Brucellosis model involving the fractional operator in (2) has a unique solution.

Proof. Proof We adopt another solution (2) say $S^{*}, E^{*}, I^{*}$, and $B^{*}$; then, we have

$$
\begin{aligned}
& S(t)-S^{*}(t)=\frac{2(1-\phi)}{(2-\phi) M(\phi)}\left[\phi_{1}(t, S(t))-\phi_{1}\left(t, S^{*}(t)\right)\right]+\frac{2 \phi}{(2-\phi) M(\phi)} \int_{0}^{t}\left[\phi_{1}(\varepsilon, S(\varepsilon))-\phi_{1}\left(t, S^{*}(\varepsilon)\right)\right] \mathrm{d} \varepsilon \\
& E(t)-E^{*}(t)=\frac{2(1-\phi)}{(2-\phi) M(\phi)}\left[\phi_{2}(t, E(t))-\phi_{2}\left(t, E^{*}(t)\right)\right]+\frac{2 \phi}{(2-\phi) M(\phi)} \int_{0}^{t}\left[\phi_{2}(\varepsilon, E(\varepsilon))-\phi_{2}\left(t, E^{*}(\varepsilon)\right)\right] \mathrm{d} \varepsilon \\
& I(t)-I^{*}(t)=\frac{2(1-\phi)}{(2-\phi) M(\phi)}\left[\phi_{3}(t, I(t))-\phi_{3}\left(t, I^{*}(t)\right)\right]+\frac{2 \phi}{(2-\phi) M(\phi)} \int_{0}^{t}\left[\phi_{3}(\varepsilon, I(\varepsilon))-\phi_{3}\left(t, I^{*}(\varepsilon)\right)\right] \mathrm{d} \varepsilon \\
& B(t)-B^{*}(t)=\frac{2(1-\phi)}{(2-\phi) M(\phi)}\left[\phi_{4}(t, B(t))-\phi_{4}\left(t, B^{*}(t)\right)\right]+\frac{2 \phi}{(2-\phi) M(\phi)} \int_{0}^{t}\left[\phi_{4}(\varepsilon, B(\varepsilon))-\phi_{4}\left(t, B^{*}(\varepsilon)\right)\right] \mathrm{d} \varepsilon
\end{aligned}
$$

By applying the norm on (23),

$$
\begin{aligned}
& S(t)-S^{*}(t) \leq \frac{2(1-\phi)}{(2-\phi) M(\phi)}\left\|\phi_{1}(t, S(t))-\phi_{1}\left(t, S^{*}(t)\right)\right\|+\frac{2 \phi}{(2-\phi) M(\phi)} \int_{0 t}^{t}\left\|\phi_{1}(\varepsilon, S(\varepsilon))-\phi_{1}\left(t, S^{*}(\varepsilon)\right)\right\| \mathrm{d} \varepsilon \\
& E(t)-E^{*}(t) \leq \frac{2(1-\phi)}{(2-\phi) M(\phi)}\left\|\phi_{2}(t, E(t))-\phi_{2}\left(t, E^{*}(t)\right)\right\|+\frac{2 \phi}{(2-\phi) M(\phi)} \int_{0}^{t}\left\|\phi_{2}(\varepsilon, E(\varepsilon))-\phi_{2}\left(t, E^{*}(\varepsilon)\right)\right\| \mathrm{d} \varepsilon \\
& I(t)-I^{*}(t) \leq \frac{2(1-\phi)}{(2-\phi) M(\phi)}\left\|\phi_{3}(t, I(t))-\phi_{3}\left(t, I^{*}(t)\right)\right\|+\frac{2 \phi}{(2-\phi) M(\phi)} \int_{0}^{t}\left\|\phi_{3}(\varepsilon, I(\varepsilon))-\phi_{3}\left(t, I^{*}(\varepsilon)\right)\right\| \mathrm{d} \varepsilon \\
& B(t)-B^{*}(t) \leq \frac{2(1-\phi)}{(2-\phi) M(\phi)}\left\|\phi_{4}(t, B(t))-\phi_{4}\left(t, B^{*}(t)\right)\right\|+\frac{2 \phi}{(2-\phi) M(\phi)} \int_{0}^{t}\left\|\phi_{4}(\varepsilon, B(\varepsilon))-\phi_{4}\left(t, B^{*}(\varepsilon)\right)\right\| \mathrm{d} \varepsilon
\end{aligned}
$$


By considering Theorems 1 and 2, we obtain the following results:

$$
\begin{aligned}
& \left\|S(t)-S^{*}(t)\right\| \leq a_{1} \frac{2(1-\phi)}{(2-\phi) M(\phi)}\left\|S(t)-S^{*}(t)\right\|+a_{1} \frac{2 \phi}{(2-\phi) M(\phi)} t\left\|S(t)-S^{*}(t)\right\|, \\
& \left\|E(t)-E^{*}(t)\right\| \leq a_{2} \frac{2(1-\phi)}{(2-\phi) M(\phi)}\left\|E(t)-E^{*}(t)\right\|+a_{2} \frac{2 \phi}{(2-\phi) M(\phi)} t\left\|E(t)-E^{*}(t)\right\|, \\
& \left\|I(t)-I^{*}(t)\right\| \leq a_{3} \frac{2(1-\phi)}{(2-\phi) M(\phi)}\left\|I(t)-I^{*}(t)\right\|+a_{3} \frac{2 \phi}{(2-\phi) M(\phi)} t\left\|I(t)-I^{*}(t)\right\|, \\
& \left\|B(t)-B^{*}(t)\right\| \leq a_{4} \frac{2(1-\phi)}{(2-\phi) M(\phi)}\left\|B(t)-B^{*}(t)\right\|+a_{4} \frac{2 \phi}{(2-\phi) M(\phi)} t\left\|B(t)-B^{*}(t)\right\| .
\end{aligned}
$$

The solution functions in (19) satisfy the following inequalities:

$$
\begin{gathered}
\left\|S(t)-S^{*}(t)\right\| \leq\left\{1-\frac{2 a_{1}}{(2-\phi) M(\phi)}(1-\phi-t \phi)\right\} \leq 0, \\
\left\|E(t)-E^{*}(t)\right\| \leq\left\{1-\frac{2 a_{2}}{(2-\phi) M(\phi)}(1-\phi-t \phi)\right\} \leq 0, \\
\left\|I(t)-I^{*}(t)\right\| \leq\left\{1-\frac{2 a_{3}}{(2-\phi) M(\phi)}(1-\phi-t \phi)\right\} \leq 0, \\
\left\|B(t)-B^{*}(t)\right\| \leq\left\{1-\frac{2 a_{4}}{(2-\phi) M(\phi)}(1-\phi-t \phi)\right\} \leq 0 .
\end{gathered}
$$

Going by the last equation, we conclude that

$$
\begin{aligned}
& S(t)=S^{*}(t), \\
& E(t)=E^{*}(t), \\
& I(t)=I^{*}(t), \\
& B(t)=B^{*}(t) .
\end{aligned}
$$

\section{Stability}

This section deals with the application of iterative Laplace transformation technique on the fractional Brucellosis model, obtaining the stability condition for the approximate solution.

4.1. Iterative Laplace Transformation Technique. We consider the Brucellosis model in (2) with the initial conditions in (3). Applying the Laplace transformation to both sides of equation (2), we have

$$
\begin{aligned}
& \frac{\rho L(S(t))-S(0)}{\rho+\phi(1-\rho)}=L(\theta-p(S+E+I)-\alpha S I-d S-\beta B S), \\
& \frac{\rho L(E(t))-E(0)}{\rho+\phi(1-\rho)}=L(\alpha S I+\beta B S-d E-\delta E), \\
& \frac{\rho L(I(t))-I(0)}{\rho+\phi(1-\rho)}=L(\delta E-d I-a I), \\
& \frac{\rho L(B(t))-B(0)}{\rho+\phi(1-\rho)}=L(n(E+I)-(\omega+c h) B) .
\end{aligned}
$$

By rearranging the following, we have

$$
\begin{aligned}
L(S(t))= & \frac{S(0)}{\rho}+\left(\frac{\rho+\phi(1-\rho)}{\rho}\right) L(\theta-p(S+E+I) \\
& -\alpha S I-d S-\beta B S), \\
L(E(t))= & \frac{E(0)}{\rho}+\left(\frac{\rho+\phi(1-\rho)}{\rho}\right) L(\alpha S I+\beta B S-d E-\delta E), \\
L(I(t))= & \frac{I(0)}{\rho}+\left(\frac{\rho+\phi(1-\rho)}{\rho}\right) L(\delta E-d I-a I), \\
L(B(t))= & \frac{B(0)}{\rho}+\left(\frac{\rho+\phi(1-\rho)}{\rho}\right) L(n(E+I)-(\omega+c h) B) .
\end{aligned}
$$

The inverse Laplace transform of (29) gives 
TABLE 1: Details of parameters [26, 46].

\begin{tabular}{lcc}
\hline Parameter & Value & Description \\
\hline$\omega$ & 3.0 & Annual natural mortality of Brucella \\
$d$ & 2.0 & Annual natural elimination rate \\
$a$ & 0.8 & Annual elimination rate for the positive cows \\
$\delta$ & 4.0 & Clinical outcome rate \\
$p$ & 1.0 & The annual birth rate of dairy cows \\
$\alpha$ & 0.2 & Indirect transmission rate \\
$\theta$ & 2 & The annual introduction number of dairy cows \\
$n$ & 0.2 & The annual quantity of Brucella \\
$c$ & 0.52 & The rate of sterilization in a disinfection \\
$h$ & 0.5 & The yearly number of disinfections \\
\hline
\end{tabular}

$$
\begin{aligned}
& S(t)=S(0)+L^{-1}\left[\left(\frac{\rho+\phi(1-\rho)}{\rho}\right) L(\theta-p(S+E+I)-\alpha S I-d S-\beta B S)\right], \\
& E(t)=E(0)+L^{-1}\left[\left(\frac{\rho+\phi(1-\rho)}{\rho}\right) L(\alpha S I+\beta B S-d E-\delta E)\right], \\
& I(t)=I(0)+L^{-1}\left[\left(\frac{\rho+\phi(1-\rho)}{\rho}\right) L(\delta E-d I-a I)\right] \\
& B(t)=B(0)+L^{-1}\left[\left(\frac{\rho+\phi(1-\rho)}{\rho}\right) L(n(E+1)-(\omega+c h) B)\right] .
\end{aligned}
$$

We obtain the following infinite series solution for the SEIB Brucellosis model in (2):

$$
\begin{aligned}
& S(t)=S_{0}+S_{1}+S_{2}+S_{3}+\cdots, \\
& E(t)=E_{0}+E_{1}+E_{2}+E_{3}+\cdots, \\
& I(t)=I_{0}+I_{1}+I_{2}+I_{3}+\cdots, \\
& B(t)=B_{0}+B_{1}+B_{2}+B_{3}+\cdots
\end{aligned}
$$

\section{Description}

In this section, we analyze the numerical simulations of the Caputo-Fabrizio Brucellosis model (2) with the following initial conditions $S_{0}=a_{1}=60, E_{0}=a_{2}=40, I_{0}=a_{3}=20$, and $B_{0}=a_{4}=10$ for specific fractional-order value $\phi \in(0,1)$. The parameters used and their values are given in Table 1 .

\section{Numerical Results and Discussion}

Figures 1-4 display the numerical results for the susceptible cows $S(t)$, exposed cows $E(t)$, infectious cows $I(t)$, and the Brucella contaminated environment $B(t)$ obtained for different values of $(\phi=1,0.9,0.8,0.7)$ with different initial conditions $S_{0}=a_{1}=60, E_{0}=a_{2}=40, I_{0}=a_{3}=20$, and $B_{0}=$ $a_{4}=10$ by utilizing the iterative Laplace transform technique of noninteger order Brucellosis model which confirms that the iterative Laplace transform method is promising and can predict the behaviour of the variables accurately within the region of consideration. The integer order has a little impact on the dynamics of the Brucellosis model. This negligible effect has been taken care of in our proposed fractional-order model and our simulations have shown that a change in the values of the order influences the transmission dynamics of the Brucellosis model. Figure 1 shows that susceptible cows $S(t)$ increase steadily in the first six months when $\phi=0.8$ but falls afterwards. However, the population of the susceptible cows falls continuously at other various fractional values of $\phi$. The plot in Figure 2 of exposed cows $E(t)$ demonstrates that the population of the exposed cow rises rapidly in the first three months when $\phi=0.8$ but drops afterwards. The population of the exposed cows also falls continuously for other fractional values of $\phi$ in Figure 2. The populations of the infectious cows $I(t)$ and the Brucella contaminated environment $B(t)$ depicted in Figures 3 and 4 , respectively, also follow similar pattern and behaviour as in Figures 1 and 2. It can be inferred that, as the fractionalorder rises from $\phi=0.5$ to $\phi=1$, the population of cows infected with Brucellosis asymptotically tends to zero.

Figures 5-8 shows different behaviours of the population of susceptible cows $S(t)$, exposed cows $E(t)$, infectious cows $I(t)$, and the Brucella contaminated environment $B(t)$, where the required simulations have been carried out for $t=12$ months while we set the value of the fractional-order parameter $\phi=1$. Furthermore, it is observed that the numerical outcome of the simulation is a function of the noninteger derivative $\phi$. This shows that the special 


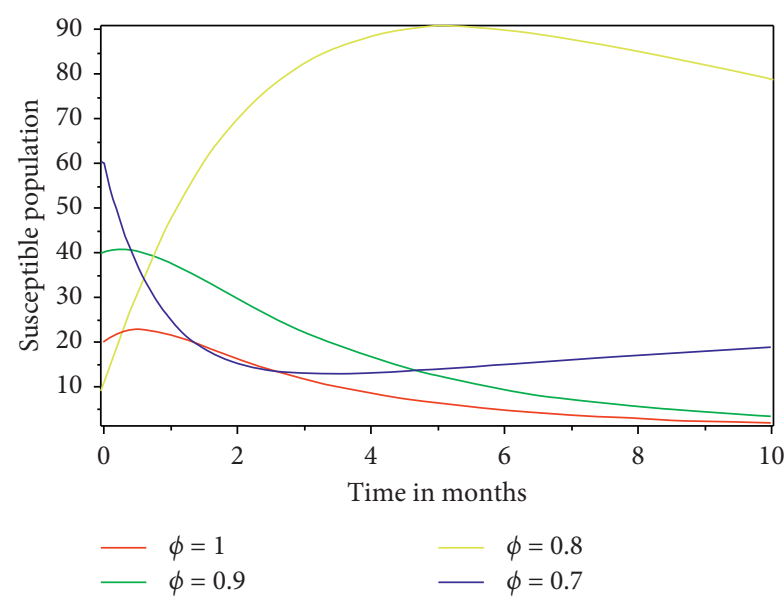

Figure 1: Approximate solution of susceptible cows.

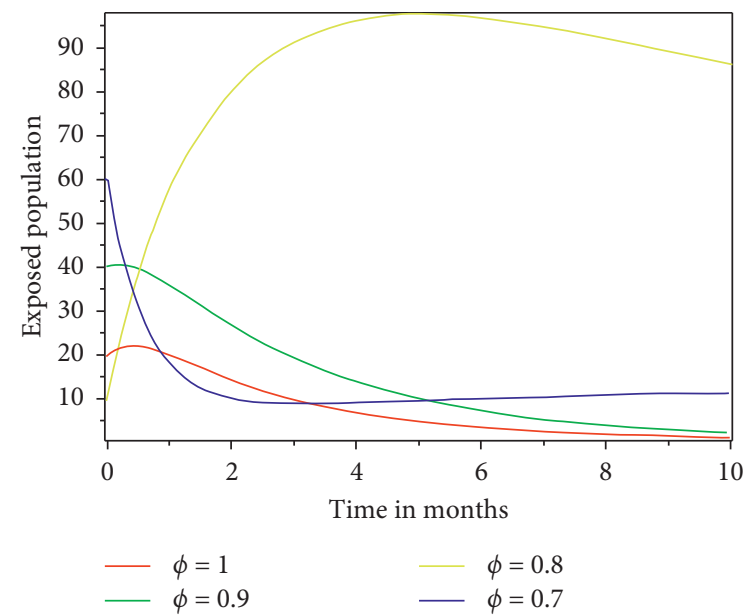

Figure 2: Approximate solution of exposed cows.

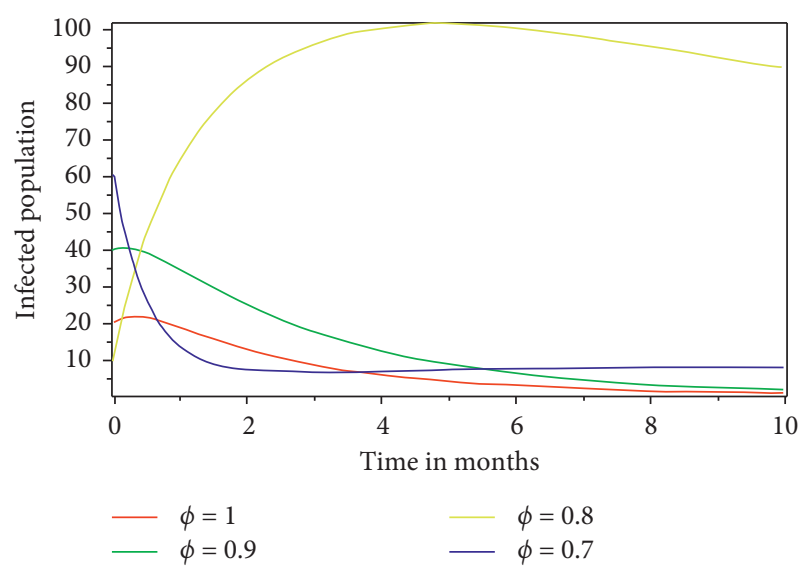

FIgURE 3: Approximate solution of infectious cows.

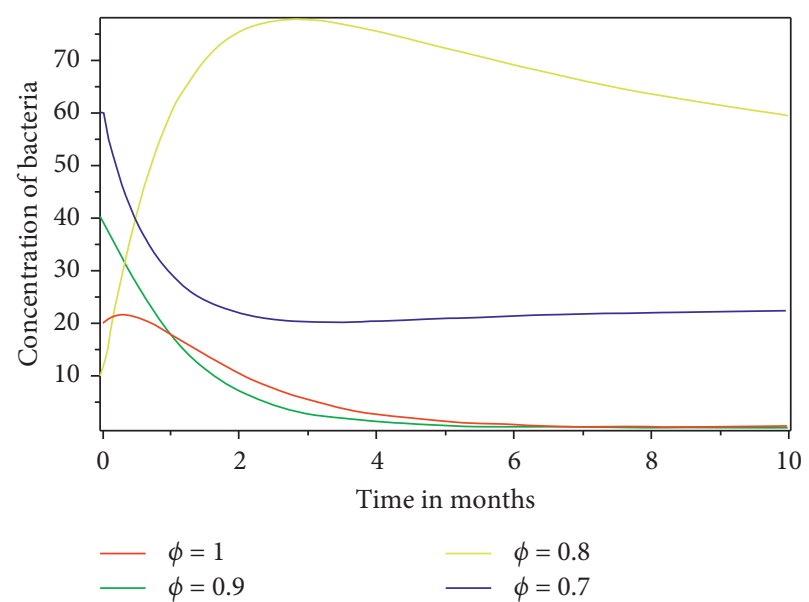

Figure 4: Approximate solution of concentration of bacteria in the environment.

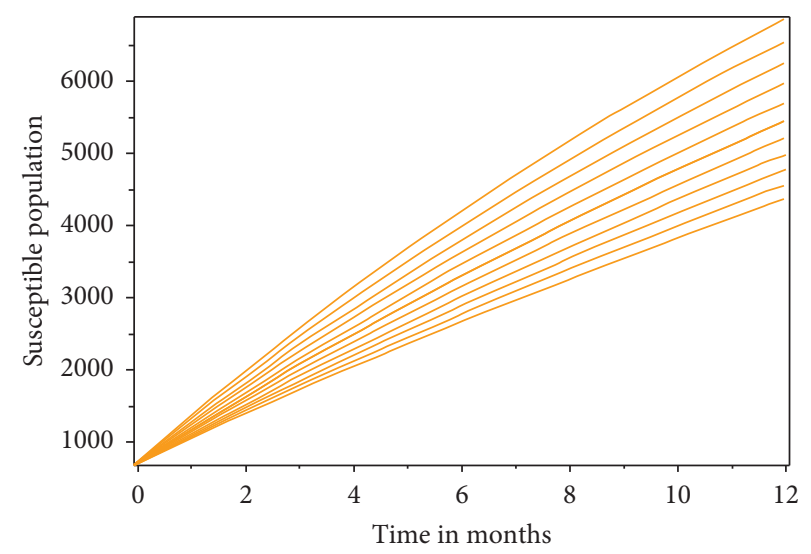

FIGURE 5: Approximate solution of susceptible cows for $\phi=1$.

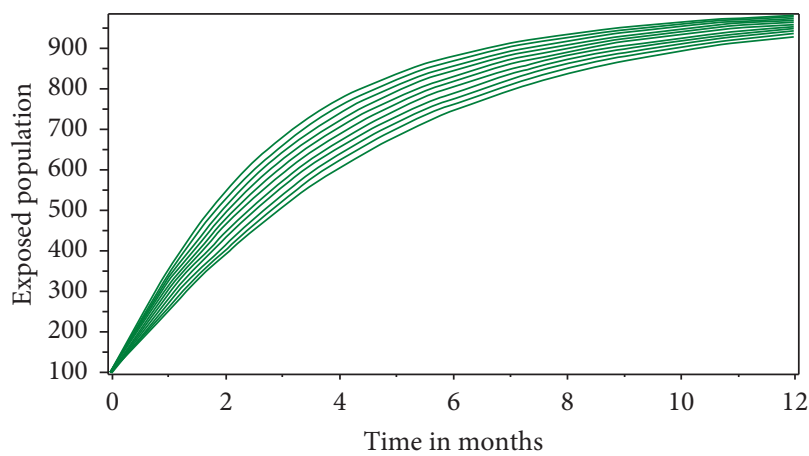

Figure 6: Approximate solution of exposed cows for $\phi=1$.

noninteger operator such as the Caputo-Fabrizio operator is efficient and plays significant roles in inaccurate predictions. Besides, the hybrid feature of Caputo-Fabrizio is strong to capture the complex dynamics and offer reliable predictions. 


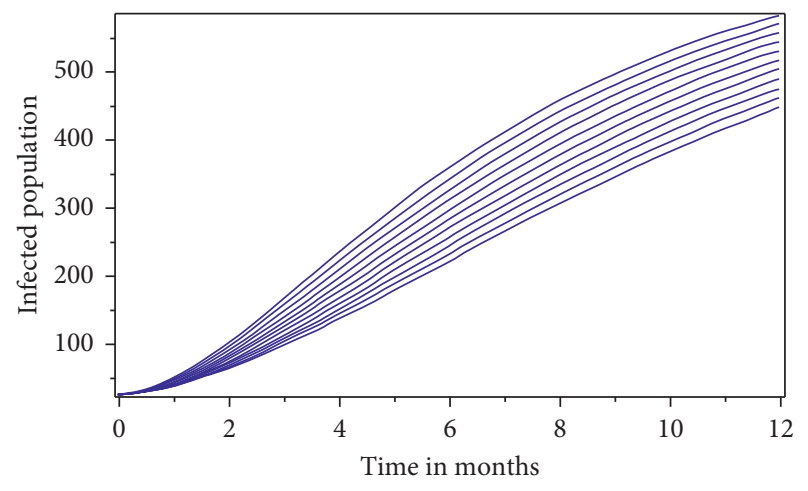

FIgURE 7: Approximate solution of infectious cows for $\phi=1$.

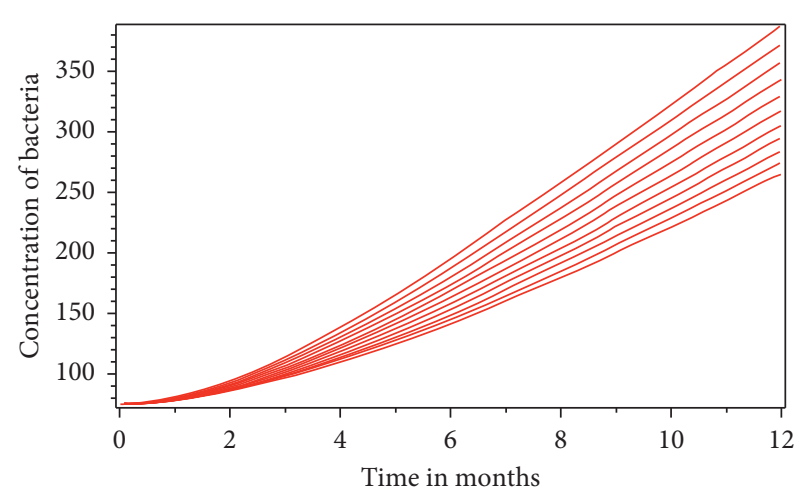

FIGURE 8: Approximate solution of concentration of bacteria for $\phi=1$.

\section{Conclusion}

In this paper, we have analyzed the Caputo-Fabrizio noninteger order Brucellosis model. Using fixed-point theorem, the steady results for the existence and uniqueness of solutions and the stability of the proposed noninteger order Brucellosis model have been derived. The iterative Laplace transform technique is applied to conduct the simulations by using a set of values whose sources are from the literature for the model parameters. The effects of various values for the order of the Caputo-Fabrizio noninteger order derivative Brucellosis model on the transmission dynamics of the disease are revealed by the results of the simulations. The results of the simulations, therefore, confirm the validity and efficiency of the Caputo-Fabrizio noninteger derivative in quantifying dynamics of Brucellosis disease and other related kind of problems.

\section{Data Availability}

The data used to support the findings of this study are available from the corresponding author upon request.

\section{Conflicts of Interest}

The author declares that they have no conflicts of interest.

\section{References}

[1] M. Li, G. Sun, G. Sun et al., "Transmission dynamics and control for a Brucellosis model in Hinggan League of Inner Mongolia, China," Mathematical Biosciences and Engineering, vol. 11, no. 5, pp. 1115-1137, 2014.

[2] T. Akhvlediani, C. T. Bautista, N. Garuchava et al., "Epidemiological and clinical features of Brucellosis in the country of Georgia," PLoS One, vol. 12, no. 1, Article ID e0170376, 2017.

[3] B. Maria-Laura, F. Vincent, and D. O'Callaghan, "Brucellosis: a worldwide zoonosis," Current Opinionin Microbiology, vol. 4, no. 1, pp. 58-64, 2001.

[4] B. G. Mantur and S. K. Amarnath, "Brucellosis in India-a review," Journal of Biosciences, vol. 33, no. 4, pp. 539-547, 2008.

[5] A. F. Brouwer, M. H. Weir, M. C. Eisenberg, R. Meza, and J. N. S. Eisenberg, "Dose-response relationships for environmentally mediated infectious disease transmission models," PLoS Computational Biology, vol. 13, no. 9, Article ID e1005765, 2017.

[6] J. Nie, G.-Q. Sun, X.-D. Sun et al., "Modeling the transmission dynamics of dairy cattle Brucellosis in Jilin Province, China," Journal of Biological Systems, vol. 22, no. 4, pp. 533-554, 2014.

[7] M.-T. Li, G.-Q. Sun, W.-Y. Zhang, and Z. Jin, "Model-based evaluation of strategies to control Brucellosis in China," International Journal of Environmental Research and Public Health, vol. 14, no. 3, p. 295, 2017.

[8] B. Ghanbari and N. Raza, "An analytical method for soliton solutions of perturbed Schrödinger's equation with quadraticcubic nonlinearity," Modern Physics Letters B, vol. 33, no. 3, Article ID 1950018, 2019.

[9] B. Ghanbari, "Abundant soliton solutions for the HirotaMaccari equation via the generalized exponential rational function method," Modern Physics Letters B, vol. 33, no. 9, Article ID 1950106, 2019.

[10] B. Ghanbari, A. Yusuf, M. Inc, and D. Baleanu, "The new exact solitary wave solutions and stability analysis for the $(2+1)$ $\$(2+1) \$$-dimensional Zakharov-Kuznetsov equation," Advances in Difference Equations, vol. 2019, no. 1, p. 49, 2019.

[11] B. Ghanbari and M. Inc, "A new generalized exponential rational function method to find exact special solutions for the resonance nonlinear Schrödinger equation," The European Physical Journal Plus, vol. 133, no. 4, pp. 133-142, 2018.

[12] B. Ghanbari, M. S. Osman, and D. Baleanu, "Generalized exponential rational function method for extended ZakharovKuzetsov equation with conformable derivative," Modern Physics Letters A, vol. 34, no. 20, Article ID 1950155, 2019.

[13] B. Ghanbari, I. Mustafa, M. Inc, and L. Rada, "Solitary wave solutions to the tzitzéica type equations obtained by a new efficient approach," Journal of Applied Analysis \& Computation, vol. 9, no. 2, pp. 568-589, 2019.

[14] M. S. Osman and B. Ghanbari, "New optical solitary wave solutions of Fokas-Lenells equation in presence of perturbation terms by a novel approach," Optik, vol. 175, pp. 328$333,2018$.

[15] M. S. Osman, B. Ghanbari, and J. A. T. Machado, "New complex waves in nonlinear optics based on complex Ginzburg-Landau equation with kerr law nonlinearity," The European Physical Journal Plus, vol. 134, no. 1, p. 20, 2019.

[16] A. Yildirima and T. Ozis, "Solution of singular ivps of LaneEmden type by homotopy perturbation method," Physics Letters A, vol. 369, no. 1-2, pp. 70-76, 2007. 
[17] J.-H. He, "The homotopy perturbation method nonlinear oscillators with discontinuities," Applied Mathematics and Computation, vol. 15, no. 1, pp. 87-292, 2004.

[18] E. Mahsa, A. Saeid, and B. Esmail, "Numerical solution of fractional partial differential equations with normalized Bernstein-wavelet method," Applications and Applied Mathematics: An International Journal, vol. 14, no. 2, pp. 890-909, 2019.

[19] S. S. Amaj and K. Sooppy Nisar, "Transmission dynamics of fractional order typhoid fever model using Caputo-Fabrizio operator," Chaos, Solitons and Fractals, vol. 128, pp. 355-365, 2019.

[20] X.-X. Zhan, C. Liu, G. Zhou et al., "Coupling dynamics of epidemic spreading and information diffusion on complex networks," Applied Mathematics and Computation, vol. 332, pp. 437-448, 2018.

[21] M.-T. Li, G.-Q. Sun, Y.-F. Wu, J. Zhang, and Z. Jin, "Transmission dynamics of a multi-group Brucellosis model with mixed cross infection in public farm," Applied Mathematics and Computation, vol. 237, no. 1, pp. 382-394, 2014.

[22] Q. Hou, X. Sun, J. Zhang, Y. Liu, Y. Wang, and Z. Jin, "Modeling the transmission dynamics of sheep Brucellosis in inner Mongolia autonomous region, China," Mathematical Biosciences, vol. 242, no. 1, pp. 51-58, 2013.

[23] G.-Q. Sun and Z.-K. Zhang, "Global stability for a sheep Brucellosis model with immigration," Applied Mathematics and Computation, vol. 246, pp. 336-345, 2014.

[24] G.-G. Jorge and R. Naulin, "Analysis of a model of bovine Brucellosis using singular perturbations," Journal of Mathematical Biology, vol. 33, no. 2, pp. 211-223, 1994.

[25] B. E. Aïnseba, C. Benosman, and P. Magal, "A model for ovine Brucellosis incorporating direct and indirect transmission," Journal of Biological Dynamics, vol. 4, no. 1, pp. 2-11, 2010.

[26] W. Zhang, J. Zhang, Y.-P. Wu, and L. Li, "Dynamical analysis of the SEIB model for Brucellosis transmission to the dairy cows with immunological threshold," Complexity, vol. 2019, Article ID 6526589, 13 pages, 2019.

[27] J. Tumwiine and G. Robert, "A mathematical model for treatment of bovine Brucellosis in cattle population," Journal of Mathematical Modeling, vol. 5, no. 2, pp. 137-152, 2017.

[28] A. A. Ayoade, M. O. Ibrahim, and O. Odetunde, "Analytical solution of a fractional order cholera model," Nigerian Journal of Scientific Research, vol. 17, no. 2, pp. 158-164, 2018.

[29] I. Ameen, "The effect of vaccination and treatment of measles disease described by a fractional order model," World $J$ of Modeling and Simulation, vol. 14, no. 1, pp. 30-38, 2018.

[30] B. R. Sontakke and A. Shaikh, "Approximate solutions of time fractional Kawahara and modified Kawahara equations by fractional complex transform," Communications in Numerical Analysis, vol. 2016, no. 2, pp. 218-229, 2016.

[31] B. Ghanbari and D. Kumar, "Numerical solution of predatorprey model with beddington-deangelis functional response and fractional derivatives with Mittag-Leffler kernel," Chaos: An Interdisciplinary Journal of Nonlinear Science, vol. 29, no. 6, Article ID 063103, 2019.

[32] B. Ghanbari and J. F. Gómez-Aguilar, "Modeling the dynamics of nutrient-phytoplankton-zooplankton system with variable-order fractional derivatives," Chaos, Solitons \& Fractals, vol. 116, pp. 114-120, 2018.

[33] B. R. Sontakke, A. S. Shaikh, and K. S. Nisar, "Approximate solutions of a generalized Hirota-Satscima coupled KDV and a coupled MKDV systems with time fractional derivatives," Malaysian Journal of Mathematical Sciences, vol. 12, no. 2, pp. 173-193, 2018.
[34] A. Shaikh, A. Tassaddiq, K. S. Nisar, and D. Baleanu, "Analysis of differential equations involving Caputo-Fabrizio fractional operator and its applications to reaction-diffusion equations," Advances in Difference Equations, vol. 2019, no. 1, p. 78, 2019.

[35] B. Ghanbari, D. Baleanu, and M. Al Qurashi, "New exact solutions of the generalized Benjamin-Bona-Mahony equation," Symmetry, vol. 11, no. 1, pp. 73-85, 2018.

[36] M. Caputo and M. Fabrizio, "A new definition of fractional derivative without singular kernel," Progress in Fractional Differentiation and Applications, vol. 1, no. 2, pp. 73-85, 2015.

[37] T. Bashiri, S. M. Vaezpour, and J. J. Nieto, "Approximating solution of fabrizio-caputo volterra's model for population growth in a closed system by homotopy analysis method," Journal of Function Spaces, vol. 2018, Article ID 3152502, 10 pages, 2018.

[38] A. Atangana and B. T. Alkahtani, "Analysis of non-homogenous heat model with new trend of derivative with fractional order," Chaos Solitons Fractals, vol. 89, pp. 566-571, 2016.

[39] A. Atangana and B. Alqahtani, "Analysis of the Keller-Segel model with a fractional derivative without singular kernel," Entropy, vol. 17, no. 12, pp. 439-4453, 2015.

[40] D. Kumar, J. Singh, D. Baleanu, and Sushila, "Analysis of regularized long-wave equation associated with a new fractional operator with Mittag-Leffler type kernel," Physica A: Statistical Mechanics and Its Applications, vol. 492, pp. 155167, 2018.

[41] M. A. Khan, Z. Hammouch, and D. Baleanu, "Modeling the dynamics of hepatitis E via the caputo-fabrizio derivative," Mathematical Modelling of Natural Phenomena, vol. 14, no. 3, p. 311, 2019.

[42] U. Saif, A. K. Muhammad, F. Muhammad, H. Zakia, and B. Dumitru, "A fractional model for the dynamics of tuberculosis infection using Caputo-Fabrizio derivative," Discrete \& Continuous Dynamical Systems-, vol. 13, no. 3, pp. 975-993, 2020.

[43] S. Jagdev, K. Devendra, H. Zakia, and A. Abdon, "A fractional epidemiological model for computer viruses pertaining to a new fractional derivative," Applied Mathematics and Computation, vol. 316, pp. 504-515, 2018.

[44] S. Qureshi and A. Yusuf, "Modeling chickenpox disease with fractional derivatives: from caputo to atangana-baleanu," Chaos, Solitons \& Fractals, vol. 122, pp. 111-118, 2019.

[45] S. Qureshi and A. Yusuf, "Fractional derivatives applied to MSEIR problems: comparative study with real world data," The European Physical Journal Plus, vol. 134, no. 4, p. 171, 2019.

[46] J. Nie, G.-Q. Sun, X.-D. Sun et al., "Modeling the transmission dynamics of dairy cattle Brucellosis in Jilin Province, China," Journal of Biological Systems, vol. 22, no. 4, pp. 533-554, 2014. 